\title{
Heart rate variability in hypoxic ischemic encephalopathy during therapeutic hypothermia
}

\author{
Robert M. Goulding ${ }^{1,2}$, Nathan J. Stevenson', Deirdre M. Murray ${ }^{1,2}$, Vicki Livingstone', Peter M. Filan², Geraldine B. Boylan ${ }^{1,2}$
}

BACKGROUND: Therapeutic hypothermia (TH) aims to ameliorate further injury in infants with moderate and severe hypoxic ischemic encephalopathy (HIE). We aim to assess the effect of TH on heart rate variability (HRV) in infants with HIE.

METHODS: Multichannel video-electroencephalography (EEG) and electrocardiography were assessed at 6-72 $\mathrm{h}$ after birth in full-term infants with HIE, recruited prior to (pre-TH group) and following (TH group) the introduction of TH in our neonatal unit. HIE severity was graded using EEG. HRV features investigated include: mean NN interval (mean NN), standard deviation of NN interval (SDNN), triangular interpolation (TINN), high-frequency $(H F)$, low-frequency (LF), very low-frequency (VLF), and LF/HF ratio. Linear mixed model comparisons were used.

RESULTS: 118 infants (pre-TH: $n=44, \mathrm{TH}: n=74$ ) were assessed. The majority of HRV features decreased with increasing EEG grade. Infants with moderate HIE undergoing TH had significantly different HRV features compared with the preTH group (HF: $P=0.016$, LF/HF ratio: $P=0.006$ ). In the pre-TH group, LF/HF ratio was significantly different between moderate and severe HIE grades $(P=0.002)$. In the TH group, significant differences were observed between moderate and severe HIE grades for SDNN: $P=0.020$, TINN: $P=0.005, \mathrm{VLF}: P=0.029$, LF: $P=0.010$, and HF: $P=0.006$.

CONCLUSION: The HF component of HRV is increased in infants with moderate HIE undergoing TH.

$\mathrm{H}$ ypoxic ischemic encephalopathy (HIE) occurs in 1-6 per 1,000 live full-term births and is caused by the interruption of blood and/or oxygen supply to the brain in the perinatal period $(1,2)$. The severity of HIE is typically determined clinically by assessing symptoms such as; altered consciousness, diminished spontaneous movements, altered muscle tone, reduced or absent primitive reflexes, seizures, problems with feeding, and manifestations of impaired autonomic function including respiratory difficulties, miosis or mydriasis, and tachycardia or bradycardia (3-5). The clinical assessment of severity, as well as other methods of assessment, such as, electroencephalography (EEG) and magnetic resonance imaging (MRI) has been associated with prognosis $(6-10)$. The role of autonomic function in the assessment of HIE, and the evidence that heart rate variability (HRV) is a measure of autonomic function, suggests that HRV may offer useful diagnostic or prognostic information in infants with HIE. Recent studies have demonstrated this prognostic value by observing an association between HRV and both short- and long-term outcome (11-14).

Therapeutic hypothermia (TH) is now standard of care for infants with moderate and severe HIE (15). Previous studies have not investigated the effect of TH on HRV in infants with moderate and severe HIE independently. These studies are important as infants with moderate and severe HIE are known to have very different long-term neurodevelopmental outcomes. This is a novel study as it investigates HRV in infants with moderate and severe HIE independently. To our knowledge, this is also the first study to compare HRV data between normothermic and hypothermic infants with HIE. This may provide a better understanding of the potential neuroprotective effect of TH in infants with moderate and severe HIE (16).

We assessed two groups of infants with HIE who were (i) monitored prior to the introduction of $\mathrm{TH}$ in our neonatal intensive care unit (NICU) (pre-TH group) and (ii) monitored following the introduction of TH in our NICU, where all infants with moderate and severe HIE received $\mathrm{TH}$ (TH group). In doing so, we aimed to (i) assess the general association between HRV and EEG grade of HIE (ii) assess the nature of this association by comparing HRV within each EEG grade of HIE (mild-moderate, mild-severe, and moderate-severe) to identify where differences are most pronounced (iii) compare HRV between pre-TH and TH groups for mild, moderate, and severe EEG grade of HIE.

\section{METHODS}

This observational study compares HRV features in infants recruited from 2003 to 2007, prior to the introduction of TH in the NICU (pre-TH group) (14) and infants recruited from 2009 to 2014, after the implementation of TH in our NICU (TH group). These were all consecutively enrolled infants at Cork University Maternity Hospital (CUMH). The CUMH was amalgamated from two maternity hospitals in 2008. With the exception of TH being introduced in the NICU, staff and neonatal care protocols were otherwise unchanged between these two recruitment periods. This is a retrospective comparison 


\section{Articles | Gouldingetal.}

study between EEG graded epochs. Data from the pre-TH group has been previously reported (14).

Term infants ( $>37 \mathrm{wk}$ gestational age) with HIE were recruited if they fulfilled $\geq 2$ of the following criteria: initial arterial $\mathrm{pH}<7.1$, Apgar score $\leq 6$ at $5 \mathrm{~min}$ after birth, initial capillary or arterial lactate level of $>7 \mathrm{mmol} / \mathrm{l}$, or abnormal neurological examination or clinical seizures. Ethical approval was obtained from the Clinical Research Ethics Committee of the Cork Teaching Hospitals. The parents of all eligible infants were approached and written informed consent was obtained. For the TH group, TH was commenced based on the TOBY trial criteria, which includes clinical evidence of encephalopathy (7).

A NicoletOne monitor (Carefusion, Madison, WI) was used to record continuous electrocardiographic (ECG) and multichannel EEG activity for up to $72 \mathrm{~h}$ after birth. The ECG and EEG signals were sampled at $256 \mathrm{~Hz}$. ECG electrodes were applied to both shoulders to record single-channel heart rate signals. EEG monitoring was used to grade the severity of HIE and for seizure management. For all EEG recordings, either silver-silver chloride or AMBU Neuroline 700 single patient surface electrodes $(70010-K / 12)$ were applied to the scalp. This EEG application follows the international 10-20 system of electrode placement (17), modified for infants, whereby, electrodes were applied at F3, F4, C3, C4, T3, T4, O1, O2, and Cz (6). A reference and a ground electrode were applied to $\mathrm{Fz}$ and the left mastoid, respectively. For this study, only infants with both good quality ECG

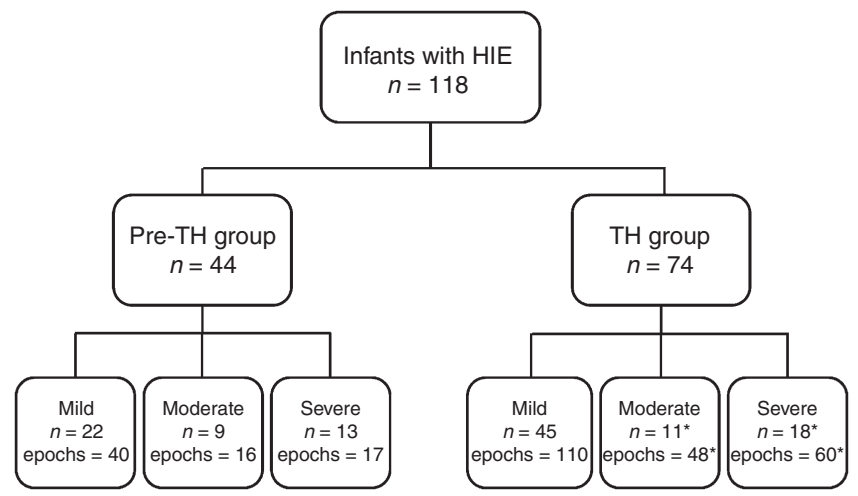

Figure 1. Flow chart describing time-points/epochs contributed by all infants in this study. HIE severity (mild/moderate/severe) is based on EEG grade; $n=$ sample size; * ${ }^{*}$ dergoing TH. HIE, hypoxic ischemic encephalopathy; EEG, electroencephalography; $\mathrm{TH}$, therapeutic hypothermia. and EEG data were included. We extracted $1 \mathrm{~h}$ epochs at six timepoints $(6,12,24,36,48$, and $72 \mathrm{~h}$ after birth). This was not always possible due to the varying quality of recordings, and as a result, each infant contributed between one and six epochs. Epochs containing EEG seizure activity were excluded. When controlling for medication we documented the administration of AEDs and morphine as yes/no.

\section{EEG Analysis}

All infants had a background EEG grade (grade 1-4) assigned to each epoch recorded at all available time-points. All epochs were retrospectively reviewed for classification. Two clinical physiologists (R.M.G. and G.B.B.) graded the EEG recordings and any inter-rater differences were discussed and a consensus achieved. This EEG grading system assesses continuity, amplitude, symmetry and synchrony, and sleep-wake cycling to assign Grade 1: Continuous background pattern with slightly abnormal activity, for example, mild asymmetry, mild voltage depression, or poorly defined sleep-wake cycling; Grade 2: Discontinuous activity with interburst interval of $<10 \mathrm{~s}$ in duration, no clear sleep-wake cycling, or clear asymmetry or asynchrony; Grade 3: Discontinuous activity with interburst interval of $10-60 \mathrm{~s}$ in duration, severe attenuation of background patterns, or no sleep-wake cycling; Grade 4: Background activity of $<10 \mu \mathrm{V}$ or severe discontinuity with interburst interval of $>60 \mathrm{~s}$ in duration (6). From these EEG grades, each epoch was categorized into three groups: mild (EEG grade 1 - normal and mildly abnormal), moderate (EEG grade 2 - moderately abnormal), or severe (EEG grade 3 and 4-severely abnormal or inactive) (14). An important point to note in the EEG analysis was that the EEG grade of HIE severity could change as the encephalopathy evolved. In addition, EEG grade of HIE represents the grade for each individual epoch rather than being an overall reflection of the infant. EEG has been used to grade the severity of HIE throughout this study.

\section{ECG Analysis}

HRV software (HRV Analysis, University College Cork, Cork) was used to identify R-peaks within the ECG signal of each $1 \mathrm{~h}$ epoch. This HRV software allows for manual correction of the identified R-peaks. This process of automated identification and manual correction of R-peaks produces an estimate of the normalized-RR interval, or NN interval. Matlab (R2013a) software was used to extract the selected HRV features from the NN interval. These HRV features were selected based on guidelines outlined in 1996 by the Task Force of the European Society of Cardiology and the North American Society of Pacing and Electrophysiology and are the same features documented in our previous HRV study (14). These features include: mean NN interval (mean NN), standard deviation of $\mathrm{NN}$ interval (SDNN),

Table 1. Summary clinical demographics and statistical comparisons for infants within the pre-TH and TH groups

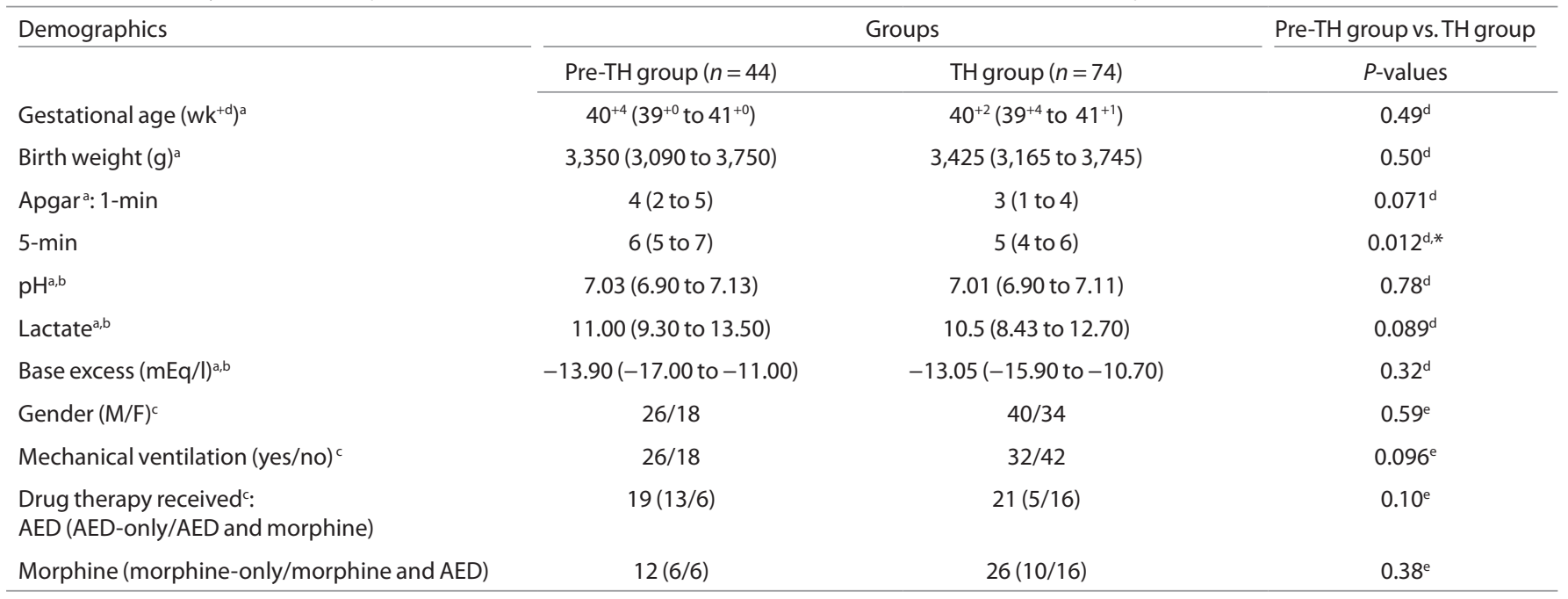

AED, antiepileptic drug; $T H$, therapeutic hypothermia.

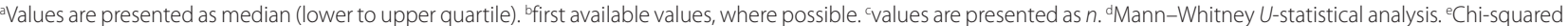

statistical test. * statistical significance $(P<0.05)$. 
triangular interpolation of NN interval histogram (TINN), the power in high-frequency (HF), low-frequency (LF), very low-frequency bands (VLF) of the NN interval, and LF/HF ratio $(14,18)$. Normalized HRV features (LFn and HFn) have been reported in previous studies (13), however, by including absolute VLF, LF, and HF values within this study, normalized features can be inferred from these for comparative purposes in the future.

Each ECG $1 \mathrm{~h}$ epoch was split into 5-min segments, with a 2.5-min overlap, for analysis. All HRV features were estimated from an interpolated $\mathrm{NN}$ interval time series. The interpolation was performed using Hermite splines and the sampling frequency was $256 \mathrm{~Hz}$. The frequency domain representation of the interpolated $\mathrm{NN}$ interval was estimated using a Periodogram (18). The following frequency bands, modified for infants, were applied: (i) VLF: 0.01-0.04 Hz; (ii) LF: $0.04-0.2 \mathrm{~Hz}$; and (iii) HF: $0.2-2 \mathrm{~Hz}$. HRV was then analyzed in relation to the EEG grade at all available time-points $(6,12,24,36,48$, and $72 \mathrm{~h}$ ). HRV features from infants contributing to the pre-TH group have been previously reported by our group (14) and are included within this study for comparative purposes.

\section{Statistical Analyses}

Statistical analyses were performed using SAS 9.3 (SAS Institute, Cary, NC) or Stata 13.0 (StataCorp LP, College Station, TX). Continuous variables were described using median (interquartile range, (IQR)) and categorical data using frequency. For comparisons between the pre-TH and TH groups, the Mann-Whitney $U$-test was used for continuous variables and the chi-squared test for categorical variables. Measures of association were calculated between HRV and EEG grade for all HIE groups (mild HIE, moderate HIE, and severe HIE) using Spearman's correlation coefficients. For each HRV feature we used an average value for each infant across their observations when their EEG grade remained the same. For infants whose EEG grade changed, the average HRV value at each EEG grade was included in the analysis; due to this averaging process, the number of values included in this analysis is different to the total number of individual epochs recorded. The equality of Spearman's correlation coefficients

Table 2. The correlation between heart rate variability features and EEG grade measured at $6-72 \mathrm{~h}$ after birth (mild EEG grade, moderate EEG grade, and severe EEG grade) in the pre-TH and TH groups

\begin{tabular}{|c|c|c|c|}
\hline Feature & $\begin{array}{l}\text { Pre-TH group } \\
\quad(n=44) \\
(\text { epochs }=50)\end{array}$ & $\begin{array}{c}\text { TH group } \\
(n=74) \\
\text { (epochs }=80)\end{array}$ & $\begin{array}{l}\text { Pre-TH vs. TH } \\
\text { comparison }^{b}\end{array}$ \\
\hline & $r^{\mathrm{a}}$ & $r^{\mathrm{a}}$ & $P$-values \\
\hline SDNN (ms) & $\begin{array}{c}-0.45 \\
(-0.65 \text { to }-0.19)^{+}\end{array}$ & $\begin{array}{c}-0.39 \\
(-0.56 \text { to }-0.19)^{\dagger}\end{array}$ & 0.697 \\
\hline TINN (ms) & $\begin{array}{c}-0.41 \\
(-0.62 \text { to }-0.15)^{\dagger}\end{array}$ & $\begin{array}{c}-0.36 \\
(-0.54 \text { to }-0.16)^{\dagger}\end{array}$ & 0.749 \\
\hline Mean VLF $\left(\mathrm{ms}^{2}\right)$ & $\begin{array}{c}-0.45 \\
(-0.65 \text { to }-0.19)^{\dagger}\end{array}$ & $\begin{array}{c}-0.28 \\
(-0.47 \text { to }-0.07)^{\dagger}\end{array}$ & 0.289 \\
\hline Mean LF $\left(\mathrm{ms}^{2}\right)$ & $\begin{array}{c}-0.41 \\
(-0.62 \text { to }-0.15)^{\dagger}\end{array}$ & $\begin{array}{c}-0.31 \\
(-0.49 \text { to }-0.09)^{\dagger}\end{array}$ & 0.535 \\
\hline Mean HF $\left(\mathrm{ms}^{2}\right)$ & $\begin{array}{c}-0.33 \\
(-0.56 \text { to }-0.06)^{+}\end{array}$ & $\begin{array}{c}-0.22 \\
(-0.42 \text { to }-0.001)^{\dagger}\end{array}$ & 0.522 \\
\hline LF/HF ratio & $\begin{array}{c}-0.17 \\
(-0.42 \text { to } 0.12)\end{array}$ & $\begin{array}{c}-0.24 \\
(-0.43 \text { to }-0.02)\end{array}$ & 0.670 \\
\hline Mean NN (ms) & $\begin{array}{c}-0.40 \\
(-0.61 \text { to }-0.14)^{\dagger}\end{array}$ & $\begin{array}{c}0.61 \\
(0.45 \text { to } 0.73)^{+}\end{array}$ & $<0.001^{*}$ \\
\hline
\end{tabular}

EEG, electroencephalography; HF, high-frequency; LF, low-frequency; mean NN, mean NN interval; SDNN, standard deviation of NN interval; TINN, triangular interpolation; $\mathrm{TH}$, therapeutic hypothermia; VLF, very low-frequency.

Spearman's rank correlation coefficient; ${ }^{\circledR}$ values are presented as correlation coefficient, $r(95 \% \mathrm{Cl}){ }^{\mathrm{b}}$ standard Fisher's z-transformation. ${ }^{\dagger} P<0.05$, correlation significantly different from $0 .{ }^{*} P<0.05$, pre-TH vs. TH comparison statistically significant result. For each infant, all contributing epochs were averaged, unless EEG grade changed between epochs, to give an assessment per infant for measures of association.
(pre-TH group and TH group) was tested using the standard Fisher's z-transformation. This equality test was performed using the Vassar Statistical Online Calculator (http://vassarstats.net/). Comparisons were made between mild, moderate, and severe EEG grades of HIE in the $\mathrm{TH}$ group using linear mixed models with a random intercept, to account for repeated measures. The variances were allowed to differ across groups if it improved model fit. Previously published results for mild, moderate, and severe comparisons in the pre-TH group are also included for comparative purposes (14). Pre-TH and TH groups were directly compared using a linear mixed model approach. For all comparisons, for each HRV feature, individual data from all epochs were included. All tests were two-sided and a $P$-value $<0.05$ was considered to be statistically significant. Epochs were categorized as mild, moderate, or severe EEG grade of HIE prior to analysis between pre$\mathrm{TH}$ and $\mathrm{TH}$ groups.

\section{RESULTS}

In total, 118 infants with HIE (pre-TH group: 44, TH group: 74) were included in the assessment of simultaneous ECG and EEG between 6 and $72 \mathrm{~h}$ after birth. We did not exclude any infant recruited due to poor quality data or artifacts. As ECG and EEG were monitored throughout recordings, interpretable data was available for at least one time-point in all infants. The correlation (correlation coefficient (95\% CI)) between EEG grade (using $24 \mathrm{~h}$ grade) and clinical assessment of HIE was 0.67 (0.36-0.97), $P<0.001$ for the pre-TH group and $0.40(0.07-0.79), P=0.02$ for the TH group. A flow chart describing all infants and data time-points ( $1 \mathrm{~h}$ epochs) contributing to this study is illustrated in Figure 1 and summary demographics for the pre-TH and TH groups are outlined in Table 1 . The first EEG epoch contributed, per infant, was available at a mean time of $12 \mathrm{~h}$ after birth in the pre-TH group and $6 \mathrm{~h}$ after birth in the TH group.

\section{HRV and Severity of HIE}

For the majority of HRV features, the correlation between EEG grade of HIE and HRV in both pre-TH and TH groups were statistically significant, being negative and low to moderate in magnitude. This indicates that there is decreased HRV with increasing EEG grade of HIE in both the pre-TH and TH groups (Table 2). This negative correlation was less pronounced in infants from the TH group, although not significantly different from the pre-TH group (Table 2). The exception to this pattern was seen when assessing mean $\mathrm{NN}$, as the pre-TH group showed a negative correlation with EEG grade of HIE, while the TH group showed a positive correlation with EEG grade of HIE $(P<0.001)$. Mean NN is a measure of heart rate and this result indicates that heart rate decreased with increasing EEG grade of HIE in infants undergoing TH (Table 2).

Analysis between EEG grades of HIE for both pre-TH and TH groups are shown in Table 3. The majority of HRV values were significantly different between mild and moderate EEG graded epochs in the pre-TH group only. In the TH group, the majority of HRV values were significantly different between the moderate and severe EEG graded epochs, and the mild and severe EEG graded epochs only (Figure 2).

\section{HRV in Pre-TH Group vs. TH Group}

There were no significant differences in HRV features between pre-TH and TH groups for mild EEG graded epochs (Table 4). 
Articles | Goulding et al.

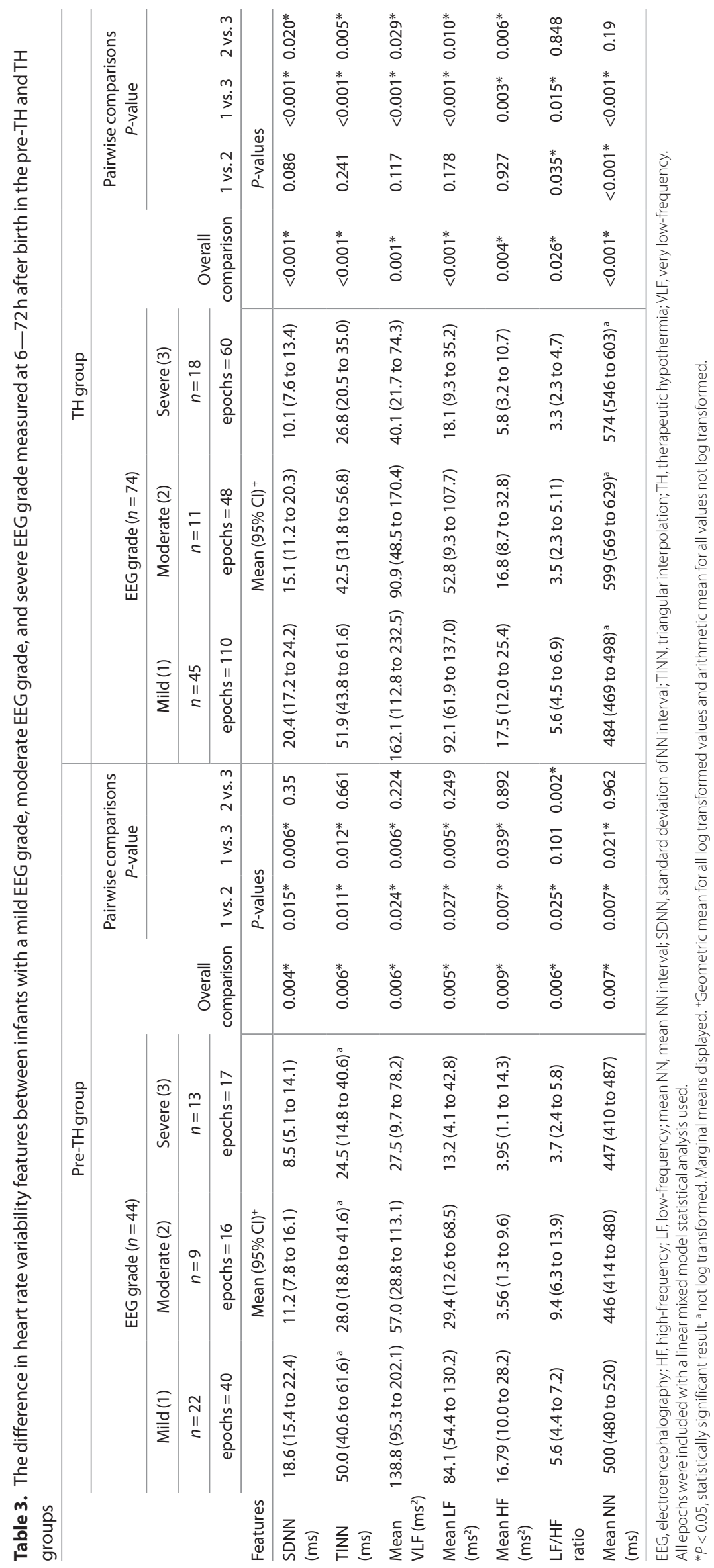




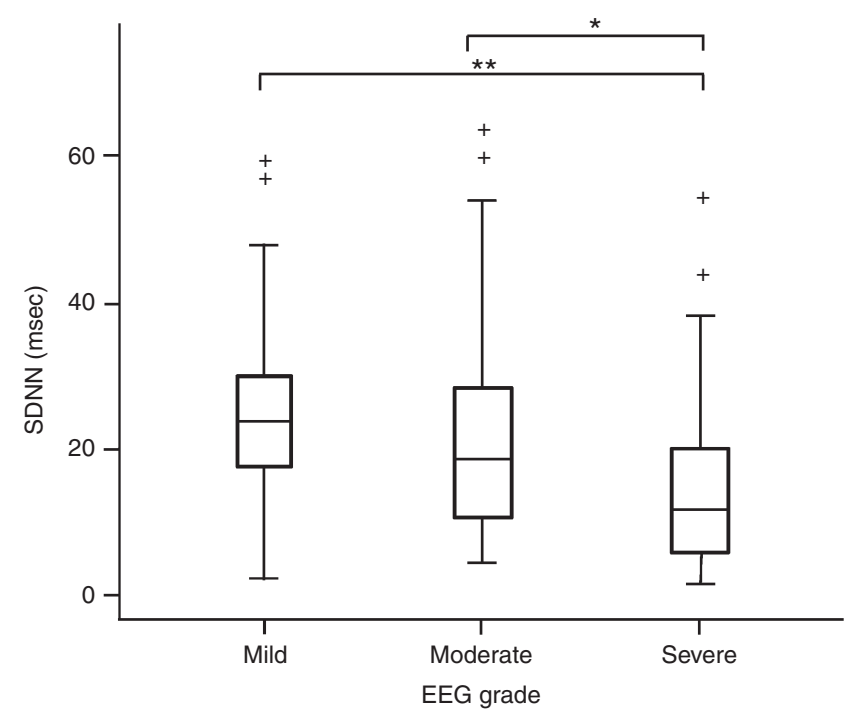

Figure 2. Plot of SDNN from the TH group for mild, moderate, and severe EEG grades. *represents statistically significant difference $(P<0.05)$; ${ }^{* *}$ represent a statistically significant difference $(P<0.001) ;{ }^{+}$represents outliers. Median values are reported with outliers identified by lower IQR-(1.5x(upper IQR-lower IQR)), or upper IQR+(1.5(upper IQR-lower $I Q R)$ ), where IQR represents interquartile range. EEG, electroencephalography; mean NN, mean NN interval; SDNN, standard deviation of NN interval; $\mathrm{TH}$, therapeutic hypothermia.

Table 4. Heart rate variability comparisons between pre-TH and TH groups for all epochs graded as mild, moderate, and severe hypoxic ischemic encephalopathy using EEG

\begin{tabular}{lccc}
\hline & \multicolumn{3}{c}{ Pre-TH group and TH group comparisons $(P$-values) } \\
\cline { 2 - 4 } Feature & $\begin{array}{c}\text { Mild EEG grade (all } \\
\text { normothermic) }\end{array}$ & $\begin{array}{c}\text { Moderate } \\
\text { EEG grade }\end{array}$ & $\begin{array}{c}\text { Severe } \\
\text { EEG grade }\end{array}$ \\
\hline SDNN (ms) & $0.44^{\mathrm{a}}$ & 0.18 & 0.71 \\
TINN (ms) & $0.89^{\mathrm{a}}$ & 0.08 & 0.83 \\
Mean VLF $\left(\mathrm{ms}^{2}\right)$ & 0.61 & 0.27 & 0.72 \\
Mean LF $\left(\mathrm{ms}^{2}\right)$ & 0.80 & 0.28 & 0.71 \\
Mean HF $\left(\mathrm{ms}^{2}\right)$ & 0.87 & $0.016^{*}$ & 0.53 \\
LF/HF ratio & $1.00^{\mathrm{a}}$ & $0.006^{\mathrm{a}, *}$ & 0.57 \\
Mean NN (ms) & $0.18^{\mathrm{a}}$ & $<0.001^{\mathrm{a}, *}$ & $<0.001^{\mathrm{a}, *}$ \\
\hline
\end{tabular}

EEG, electroencephalography; HF, high-frequency; LF, low-frequency; NN, mean; SDNN, standard deviation of NN interval; $T I N N$, triangular interpolation; $T H$, therapeutic hypothermia; VLF, very low-frequency.

$P$-values are reported. Linear mixed model statistical analysis.

a not log transformed for statistical comparisons. ${ }^{*} P<0.05$, statistically significant result.

In infants with moderate EEG graded epochs undergoing $\mathrm{TH}$, the HF feature of HRV was increased $(P=0.016)$ (Figure 3a) with a resultant decrease in the LF/HF ratio $(P=0.006)$ and the mean NN was significantly increased $(P<0.001)$ (Figure $3 \mathbf{b}$ ) (Table 4). In infants with severe EEG graded epochs, LF and HF were unchanged whereas only the mean $\mathrm{NN}$ was significantly increased in the TH group $(P<0.001$; Table 4$)$.

\section{Medication}

There were no significant differences for antiepileptic drugs (AED) and morphine administration when comparing between pre-TH and TH groups (Table 1). Our unit protocol for the use of inotropic support did not vary between recruitment periods and, in total, four infants (pre- $\mathrm{TH}=2$ and $\mathrm{TH}$ $=2$, all with severe EEG grades) received inotropic support. Echocardiographic studies and cardiac enzymes were not assessed as part of the study protocol.

\section{DISCUSSION}

We compared HRV features in infants with HIE from pre-TH and TH eras. HRV features were significantly associated with EEG grade of HIE in both the pre-TH and TH groups; with a reduction in HRV seen as HIE severity increased, for the majority of features. Reduced HRV is associated with increasing subcortical neuronal injury or an inability of the damaged heart to respond to the demands of the autonomic nervous system $(19,20)$. Within the TH group, the negative association between severity of EEG grade of HIE and HRV features is less pronounced, although still significant. This may be due to the preserved HRV in infants with moderate HIE undergoing TH.

There is a significant association between HIE severity and neurodevelopmental outcome (21); infants with a moderate EEG grade of HIE are more likely to have abnormal neurodevelopmental outcome compared with infants who have a mild EEG grade of HIE $(6,7)$. Since the introduction of TH in recent years, the outcome of infants with moderate HIE has improved (16). The correlation between HRV and outcome was not assessed as part of this study, however, our results do provide support to an altered evolution of disease during the secondary phase of injury for infants with moderate HIE undergoing TH. The significant difference in HRV (SDNN, TINN, VLF, LF, and HF) seen between mild and moderate EEG grades of HIE in the pre-TH group is no longer evident in the TH group and, instead, a significant difference is now seen between moderate and severe EEG grades of HIE (SDNN, TINN, VLF, LF, and HF); and mild and severe EEG grades of HIE (SDNN, TINN, VLF, LF, HF, LF/HF ratio, and mean NN) in the $\mathrm{TH}$ group. This implies that autonomic function may be improved in infants with a moderate EEG grade undergoing TH. HRV is associated with function of subcortical rather than cortical brain regions $(19,22,23)$ and our findings of preserved HRV in infants with moderate HIE undergoing TH support several observational magnetic resonance imaging studies, which have shown a reduction in subcortical lesions in infants undergoing TH (19,22-24). Studies reporting reduced subcortical lesions in TH are further demonstrated through increased HF and decreased LF/ HF ratio seen in infants with a moderate EEG grade of HIE in the TH group compared with the pre-TH group. This was most pronounced in the HF feature of HRV. HF is thought to represent parasympathetic activity of the autonomic nervous system (25) and increased parasympathetic function is associated with neuroprotection $(26,27)$. We hypothesize that this increase in the HF component of HRV may be a manifestation of the neuroprotective effect of $\mathrm{TH}$.

We have shown that HRV is associated with the severity of HIE in full-term infants treated with TH. This finding is 

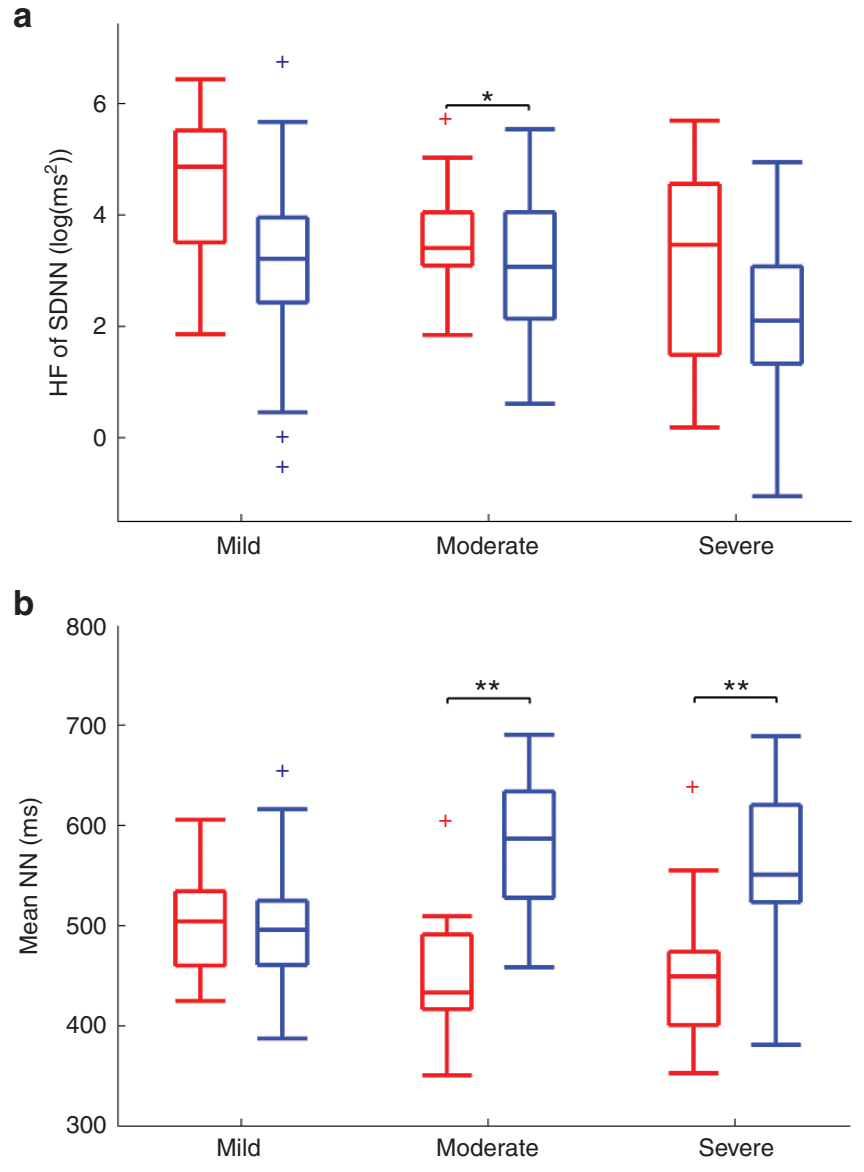

Figure 3. Boxplot of (a) the HF and (b) mean NN features between pre-TH and TH groups for all EEG grades of HIE (mild, moderate, and severe). ${ }^{*}$ represents a statistically significant difference of $P<0.05$; **represents a statistically significant difference of $P<0.001 ;{ }^{+}$represents outliers. Median values are reported with outliers identified by lower IQR-(1.5(upper IQR-lower IQR)), or upper IQR+(1.5(upper IQR-lower IQR)), where IQR represents interquartile range. Pre-TH group represented by color Red and TH group represented by color Blue. EEG, electroencephalography; HIE, hypoxic ischemic encephalopathy; $\mathrm{HF}$, high-frequency; mean $\mathrm{NN}$, mean $\mathrm{NN}$ interval; $\mathrm{TH}$, therapeutic hypothermia.

supported by previous literature (11-13). Differences between these studies include the HRV features assessed and the amalgamation of HIE groups for their assessment. The advantage of our study is that we have compared HRV features between normothermic and hypothermic infants with HIE.

We have shown that changes in autonomic function, as measured by HRV, can help grade the severity of HIE in normothermic and hypothermic infants (14). In contrast to clinical observations of increased parasympathetic function (via miosis) and bradycardia, in normothermic infants with a moderate EEG grade of HIE, we see an increase in heart rate and decrease in parasympathetic function (via the HF feature of HRV) (5). Differences in parasympathetic function may be exacerbated by the methods of measurement, as parasympathetic function is not the sole cause of miosis and HF.

Mean NN was significantly increased in moderate and severe EEG graded epochs from the TH group compared with moderate and severe EEG graded epochs from the pre-TH group.
This was expected as hypothermia has been shown to reduce energy and metabolic demand in animal studies and, therefore, decrease heart rate (28). We do not expect the increase in mean $\mathrm{NN}$ in hypothermia to have influenced the reported changes in HRV within this study, as we did not see a consistent change across all HRV features between the pre-TH and TH groups.

The autonomic nervous system is not the only factor that influences HRV; there are several possible confounding factors that may affect the interpretation of HRV results in this study. Drug administration is a potential confounding factor, though phenobarbitone (our first-line AED) administration has not been associated with changes in HRV in infants with HIE (12) and in our study differences in the administration of AEDs or morphine between the pre-TH and TH groups were not seen. The inclusion of echocardiographs or cardiac enzyme data in future studies would further strengthen our findings as any HRV alterations related to cardiac dysfunction rather than neurological dysfunction could be assessed. It is possible that different recruitment periods between the pre-TH and TH groups may have introduced a confounder due to variations in the clinical care of infants with moderate and severe HIE, though we were not aware of any such confounders. This is supported by the fact that there were no significant differences in HRV features between the pre-TH and TH groups for infants with mild EEG grades of HIE suggesting homogeneity between the pre- $\mathrm{TH}$ and $\mathrm{TH}$ groups (infants with mild HIE were not treated with TH). Differences in time after birth between groups may have also influenced our results. However, EEG grade is independent of time and we have directly compared HRV features with EEG grade at specific time-points after birth. We have also used the same time-points in pre-TH and $\mathrm{TH}$ groups to reduce any confounding effect.

\section{CONCLUSION}

The association between EEG grade and HRV remains in infants with HIE in the era of TH, however, the nature of this association has changed. Infants with a moderate EEG grade of HIE undergoing TH have increased HRV with specific increases seen in HRV components related to parasympathetic control.

\section{ACKNOWLEDGMENTS}

The authors acknowledge the assistance of Denis Dwyer, UCC software engineer, in developing the HRV software, the assistance of Brian Walsh and Evonne Low, Research Fellows, in collecting and recording data in the TH group, and staff from the NICU of Cork University Maternity Hospital for their assistance and support throughout the recruitment period.

\section{STATEMENT OF FINANCIAL SUPPORT}

This research was supported by a Science Foundation Ireland Principal Investigator Award (SFI 10/IN.1/B3036) and a Science Foundation Ireland Centers Programme (12/RC/2272).

Disclosure: All authors have no financial ties to products in the study and no potential or perceived conflicts of interest.

\section{REFERENCES}

1. Shankaran S. Neonatal encephalopathy: Treatment with hypothermia. J Neurotrauma 2009;26:437-43.

2. Johnston MV. Neonatal Hypoxic-Ischemic Brain Insults and their Mechanisms. In: New Concepts in Cerebral Ischemia. New York: CRC Press, 2001:33-51. 
3. Graham EM, Ruis KA, Hartman AL, Northington FJ, Fox HE. A systematic review of the role of intrapartum hypoxia-ischemia in the causation of neonatal encephalopathy. Am J Obstet Gynecol 2008;199:587-95.

4. Hankins GD, Speer M. Defining the pathogenesis and pathophysiology of neonatal encephalopathy and cerebral palsy. Obstet Gynecol 2003;102: 628-36.

5. Sarnat HB, Sarnat MS. Neonatal encephalopathy following fetal distress. A clinical and electroencephalographic study. Arch Neurol 1976;33:696-705.

6. Murray DM, Boylan GB, Ryan CA, Connolly S. Early EEG findings in hypoxic-ischemic encephalopathy predict outcomes at $2 \mathrm{y}$. Pediatrics 2009;124:e459-67.

7. Azzopardi D, Brocklehurst P, Edwards D, et al.; TOBY Study Group. The TOBY Study. Whole body hypothermia for the treatment of perinatal asphyxial encephalopathy: A randomised controlled trial. BMC Pediatr 2008;8:17.

8. Jacobs SE, Morley CJ, Inder TE, et al.; Infant Cooling Evaluation Collaboration. Whole-body hypothermia for term and near-term newborns with hypoxic-ischemic encephalopathy: A randomized controlled trial. Arch Pediatr Adolesc Med 2011;165:692-700.

9. Shankaran S, Laptook AR, Ehrenkranz RA, et al.; NICHD Neonatal Research Network. Whole-body hypothermia for neonates with hypoxicischemic encephalopathy. N Engl J Med 2005;353:1574-84.

10. Shankaran S, Pappas A, McDonald SA, et al.; Eunice Kennedy Shriver NICHD Neonatal Research Network. Childhood outcomes after hypothermia for neonatal encephalopathy. N Engl J Med 2012;366:2085-92.

11. Massaro AN, Govindan RB, Al-Shargabi T, et al. Heart rate variability in encephalopathic newborns during and after therapeutic hypothermia. J Perinatol 2014;34:836-41.

12. Vergales BD, Zanelli SA, Matsumoto JA, et al. Depressed heart rate variability is associated with abnormal EEG, MRI, and death in neonates with hypoxic ischemic encephalopathy. Am J Perinatol 2014;31:855-62.

13. Aliefendioğlu D, Doğru T, Albayrak M, Dibekmısırlığlu E, Sanlı C. Heart rate variability in neonates with hypoxic ischemic encephalopathy. Indian J Pediatr 2012;79:1468-72.

14. Goulding RM, Stevenson NJ, Murray DM, Livingstone V, Filan PM, Boylan GB. Heart rate variability in hypoxic ischemic encephalopathy: Correlation with EEG grade and 2-y neurodevelopmental outcome. Pediatr Res 2015;77:681-7.
15. Gancia P, Pomero G. Therapeutic hypothermia in the prevention of hypoxic-ischaemic encephalopathy: New categories to be enrolled. J Matern Fetal Neonatal Med 2012;25 Suppl 4:94-6.

16. Jacobs SE, Berg M, Hunt R, Tarnow-Mordi WO, Inder TE, Davis PG. Cooling for newborns with hypoxic ischaemic encephalopathy. Cochrane Database Syst Rev 2013;Issue 1:19-20.

17. Malmivuo J, Plonsey R. Electroencephalography. In: Bioelectromagnesium: Principles and Applications of Bioelectric and Biomagnetic Fields. New York: Oxford University Press, 1995:258-60.

18. Malik M. Heart rate variability: Standards of measurement, physiological interpretation, and clinical use. Circulation 1996;93:1043-65.

19. de Vries LS, Groenendaal F. Patterns of neonatal hypoxic-ischaemic brain injury. Neuroradiology 2010;52:555-66.

20. Fabiani R, Ceconi C, Curello S, Alfieri O, Visioli O. Myocardial damage during ischaemia and reperfusion. Eur Heart J 1993;14 Suppl G:25-30.

21. Ahearne CE, Boylan GB, Murray DM. Short and long term prognosis in perinatal asphyxia: An update. World J Clin Pediatr 2016;5:67-74.

22. Novak V, Novak P, deMarchie M, Schondorf R. The effect of severe brainstem injury on heart rate and blood pressure oscillations. Clin Auton Res 1995;5:24-30.

23. George S, Gunn AJ, Westgate JA, Brabyn C, Guan J, Bennet L. Fetal heart rate variability and brain stem injury after asphyxia in preterm fetal sheep. Am J Physiol Regul Integr Comp Physiol 2004;287:R925-33.

24. Rutherford M, Ramenghi LA, Edwards AD, et al. Assessment of brain tissue injury after moderate hypothermia in neonates with hypoxic-ischaemic encephalopathy: A nested substudy of a randomised controlled trial. Lancet Neurol 2010;9:39-45.

25. Malliani A, Pagani M, Lombardi F, Cerutti S. Cardiovascular neural regulation explored in the frequency domain. Circulation 1991;84:482-92.

26. Cheyuo C, Jacob A, Wu R, Zhou M, Coppa GF, Wang P. The parasympathetic nervous system in the quest for stroke therapeutics. J Cereb Blood Flow Metab 2011;31:1187-95.

27. Ay I, Sorensen AG, Ay H. Vagus nerve stimulation reduces infarct size in rat focal cerebral ischemia: An unlikely role for cerebral blood flow. Brain Res 2011;1392:110-5.

28. Liu X, Tooley J, Løberg EM, Suleiman MS, Thoresen M. Immediate hypothermia reduces cardiac troponin I after hypoxic-ischemic encephalopathy in newborn pigs. Pediatr Res 2011;70:352-6. 